\title{
Maximal slicings in spherical symmetry: local existence and construction
}

\author{
Isabel Cordero-Carrión ${ }^{1}$, José María Ibáñez ${ }^{2}$ \\ and Juan Antonio Morales-Lladosa ${ }^{3}$
}

\begin{abstract}
We show that any spherically symmetric spacetime locally admits a maximal spacelike slicing and we give a procedure allowing its construction. The construction procedure that we have designed is based on purely geometrical arguments and, in practice, leads to solve a decoupled system of first order quasi-linear partial differential equations. We have explicitly built up maximal foliations in Minkowski and Friedmann spacetimes. Our approach admits further generalizations and efficient computational implementation. As by product, we suggest some applications of our work in the task of calibrating Numerical Relativity complex codes, usually written in Cartesian coordinates.
\end{abstract}

PACS: 04.20.Cv, 04.20.-q, 04.20.Ex, 04.25.D-, 02.30.Jr, 02.60.Lj

${ }^{1}$ Max-Planck-Institute for Astrophysics, Garching, Karl-Schwarschild-Str. 1, D-85741 Garching, Germany E-mail: chabela@mpa-garching.mpg.de

2 Departament d'Astronomia i Astrofísica, Universitat de València, E-46100 Burjassot, València, Spain. E-mail: Jose.M. Ibanez@uv.es

${ }^{3}$ Departament d'Astronomia i Astrofísica, Universitat de València, E-46100 Burjassot, València, Spain. E-mail: antonio.morales@uv.es 


\section{Introduction}

In Lorentzian geometry, a maximal hypersurface is one that is spacelike and has vanishing mean extrinsic curvature, $K=0, K$ being the trace of the extrinsic curvature of the hypersurface. The name comes from the fact that the induced volume functional reaches a local maximum with respect the variations that keep fixed a given boundary. Maximal hypersurfaces were considered by Lichnerowicz ${ }^{1}$ to solve Einstein's constraint equations, giving motivation for subsequent studies on the subject.23/4/5/6/7 In fact, the existence of maximal hypersurfaces is extensively used in Mathematical Relativity $\stackrel{8}{[}$ This property is a very simple geometric assumption allowing one to establish general results for a very large variety of spacetimes, for instance, local or asymptotically stationary or conformally flat spacetimes.

In this paper, we will use the term maximal slicing when referring to a (non intersecting) family of spacelike maximal hypersurfaces which locally foliates a certain domain of spacetime. This type of slicing has very nice properties as, for example: i) the well-known singularity avoidance capability, 9 ii) it is well adapted to the propagation of gravitational

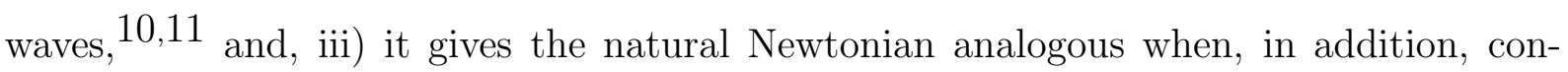
formal flatness is imposed on each slice. 12 Maximal slicing condition has been recently used in the Fully Constrained Formulation of Einstein equations derived by the Meudon group, $\frac{1314}{\text { in the field of Numerical Relativity. } 15 \mid 16}$ The study of maximal hypersurfaces is not only interesting to carry out practical applications, but also, to solve fundamental questions in Relativity. Consider, for example, the proof of the positiveness of the mass for isolated gravitating systems, that was firstly proved by assuming the existence of a maximal spacelike hypersurface, $\frac{17}{17}$ and only then, it was finally established by removing this reasonable hypothesis. 18

The study of maximal slicings has been mainly motivated by the resolution of the initial value problem. ${ }^{1}$ This idea was popularized by Smarr and York ${ }^{9[19}$ Then, for a given class of spacetimes, the construction of a family of slices with a given property (e.g., maximal slicing and/or conformal flatness) may be dynamically accomplished in an evolution scheme: assuming the existence of an initial 3-surface with that property, Einstein equations are used to evolve locally the corresponding additional constraint.

This strategy can be applied both analytically and numerically, as it has been done by 
Estabroook et al.20 and by Beig and Murchadha ${ }^{21}$ to construct maximal slicings in the extended Schwarzschild geometry. This maximal foliation of the Kruskal-Szekeres black hole was obtained independently by Reinhart ${ }^{22}$ from a different approach. Reinhart's procedure 22 does not make any use of Einstein equations, being essentially a geometrical (non dynamical) approach.

Similar examples of maximal slicings have been examined by Malec 23 in the ReissnerNordström geometry, and by Petrich et al. 24 in dynamical spherically symmetric spacetimes (SSSTs) of dust collapse scenarios, by using isotropic coordinates. Eardley and Smarr 25 obtained general results and presented some conjectures about the existence of Cauchy time functions whose level surfaces are maximal (or foliations with constant mean extrinsic curvature) with direct applications to the Lemaitre-Tolman-Bondi geometry. Of course, the study of the existence (and uniqueness) of other types of foliations deserves a lot of interest in SSSTs and more general spacetimes. That is the case of synchronizations by flat instants (Painlevé-Gullstrand slicings) and their generalizations. 26|27|28|29|30|31|32 Recently, foliations with constant mean curvature have been constructed by Malec ${ }^{33}$ in the Schwarzschild geometry.

In spite of their extended use, the existence of maximal slicings in SSSTs has been only established for vacuum and for some particular energy contents. $12|20| 21|22| 23|24| 25$ There is, as far as we know, no theorem stating that always it is possible to build a maximal slicing in a SSST.

In this work, we aim to prove the local existence of maximal slicings in an arbitrary SSST. We will follow a purely geometrical approach, independent of Einstein equations, complementary to the standard time evolution strategy $12|20| 21|23| 24 \mid 25$ Although our study is independent of the field equations, one by-product of our approach, which could be of interest in the field of Numerical Relativity, is that it provides a means to assess complex and sophisticated 3D numerical codes built to solve Einstein equations. Indeed, most of the current 3D present-day Numerical Relativity codes are written in Cartesian coordinates. Two classical, standard test-beds in spherical symmetry are the long-term stable evolution of a Schwarzschild black hole (vacuum), and the Oppenheimer-Snyder collapse $^{34}$ of dust (matter). In both cases the builder of a Numerical Relativity code aims at the recovering of the corresponding well-known solutions in spherical symmetry. This is an obvious assessment (in the 3D case) of a code. Let us consider the Oppenheimer-Snyder 
analysis of the collapse of a ball of dust; it is the simplest approach to the current studies on the complex phenomena of the stellar core collapse (in the astrophysical scenario of hydrodynamical supernovae). Hence, for a given 3D numerical code, written in Cartesian coordinates, the Oppenheimer-Snyder solution is a nice test-bed to asses the performances of that code, but also to calibrate it against any spurious gravitational radiation released as a consequence of grid effects. Since our approach provides a procedure to generate a maximal slicing in a SSST, this, in practice, means that any SSST, not necessarily satisfying the maximal slicing condition, can be reformulated in terms of this slicing and used to check a numerical 3D solution generated by a code written in Cartesian coordinates or not and satisfying the maximal slicing gauge.

Our analysis has focused on SSSTs in standard coordinates. An intrinsic characterization of SSSTs in terms of metric tensorial concomitants has been very recently accomplished by Ferrando and Sáez. ${ }^{35}$ The study of SSSTs is of particular interest in different domains of general relativity: classification of exact solutions of Einstein equations, simplified models of astrophysical and cosmological scenarios, $\frac{36}{,}$ test-bed solutions for fully general-relativistic time-dependent numerical codes which evolve matter in strong gravitational fields.24/37/38/39 SSSTs provide the background for a perturbative theory. All these reasons advise us to start our analysis in such a simple symmetry and to defer in a future paper its extension to less strongly symmetric spacetimes.

The paper is organized as follows. In Sec. 2 we consider (in a SSST) vorticity-free and non-expanding observers, that is, those that are orthogonal to maximal slicings. In Sec. 3 we analyze the existence of a coordinate transformation allowing a new coordinate time that has associated a maximal slicing. In Sec. 4 we derive a decoupled system of three first order partial differential equations that proves the local existence of a maximal slicing and, in Sec. 5, we provide a general procedure allowing its construction. This procedure is illustrated in Sec. [6 considering the most simple geometry: a flat spacetime, solving the problem analytically. The procedure is also illustrated in Sec. 7 considering a closed Friedmann spacetime, which is used in physical applications of dust collapse. Finally, in Sec. 8, we summarize the results. It will be understood that the title of the sections always concern spacetimes with spherical symmetry. A short communication $\underline{40}$ of preliminary results of this work was presented at the Spanish Relativity meeting ERE-2009. 


\section{Kinematical approach to maximal slicings}

Without loss of generality, we start with the canonical form of the metric of a SSST:

$$
d s^{2}=A d t^{2}+2 C d t d r+B d r^{2}+D d \Omega^{2}
$$

(see, e.g. the book by Plebański and Krasiński ${ }^{36}$ ) where $d \Omega^{2}=d \theta^{2}+\sin ^{2} \theta d \varphi^{2}$ is the metric of the 2-sphere, and $A, B, C, D$ are smooth functions of $t$ and $r$ such that $A B-C^{2}<0$, which guarantees the Lorentzian character of the metric. In addition we choose the signature $(-,+,+,+)$, and accordingly $D>0$. Partial derivatives with respect to $r$ and

$t$ will be denoted as $\frac{\partial f}{\partial r}=f^{\prime}$ and $\frac{\partial f}{\partial t}=\dot{f}$ respectively. The spatial metric $\gamma_{i j}$ induced on the hypersurfaces $\Sigma_{t}$, defined by $t=$ constant, is $\gamma_{i j}=\operatorname{diag}\left(B, D, D \sin ^{2} \theta\right)$, where $B>0$ since we are considering spacelike hypersurfaces. Let $n=n^{\mu} \partial_{\mu}$ be the future pointing timelike unit normal to the hypersurfaces $\Sigma_{t}$,

$$
n=\frac{1}{\alpha}\left(\frac{\partial}{\partial t}-\frac{C}{B} \frac{\partial}{\partial r}\right), \alpha=\sqrt{\frac{C^{2}}{B}-A} .
$$

The mean extrinsic curvature $K$ of $\Sigma_{t}$ is

$$
-K=\nabla_{\mu} n^{\mu}=\frac{1}{D \sqrt{C^{2}-A B}}\left[(\sqrt{B} D)^{\bullet}-\left(\frac{C D}{\sqrt{B}}\right)^{\prime}\right],
$$

where $\nabla$ is the covariant derivative with respect to the spacetime metric given by Eq. (11), and $\mu \in\{t, r, \theta, \varphi\}$. Simplifying this relation, we obtain

$$
K=\frac{1}{2 \alpha B}\left(-\dot{B}-2 B \frac{\dot{D}}{D}+2 C^{\prime}-C \frac{B^{\prime}}{B}+2 C \frac{D^{\prime}}{D}\right) .
$$

That the surfaces $t=$ constant are maximal means that the maximal slicing condition $K=0$ is fulfilled. In the following, we assume that $A, B, C$ and $D$ are known functions. We aim to prove the local existence of a maximal slicing in spherical symmetry. From a kinematical point of view, we look for the existence of a non-expanding observer $u$ on the 2 -surfaces defined by constant $\theta$ and $\varphi$, i.e., a unit and future directed radial timelike field $u=u^{\mu}=\left(u^{t}(t, r), u^{r}(t, r), 0,0\right)$, such that $\nabla_{\mu} u^{\mu}=0$ or, equivalently,

$$
\left(u^{t} D \sqrt{C^{2}-A B}\right)^{\cdot}+\left(u^{r} D \sqrt{C^{2}-A B}\right)^{\prime}=0 .
$$

The condition $g(u, u)=-1$, with respect to the spacetime metric $g_{\mu \nu}$ given by Eq. (11), leads to the following relation between the components of $u$ :

$$
A\left(u^{t}\right)^{2}+B\left(u^{r}\right)^{2}+2 C u^{t} u^{r}=-1 \text {. }
$$


Eq. (66) allows to express $u^{r}$ in terms of $u^{t}$ and the metric components when the associated discriminant is not negative, that is,

$$
\left(u^{t}\right)^{2} \geq \frac{B}{C^{2}-A B}
$$

Substituting the expression for $u^{r}$ in Eq. (5), one obtains a first order partial differential equation for $u^{t}$. In order to guarantee the existence of a maximal slicing, the solutions of this equation have to satisfy the above algebraic constraint (7) on $u^{t}$.

In the next section, we introduce a change of coordinates $\{\tilde{t}=\tilde{t}(t, r), \tilde{r}=\tilde{r}(t, r), \theta, \varphi\}$ and we require that the new coordinate hypersurfaces $\tilde{t}=$ constant be maximal. As we show below, no additional algebraic constraint must be considered when the conditions assuring the existence of this coordinate transformation are properly taken into account.

\section{Main equations of maximal slicings}

According to the previous comment, we need to introduce two fields, $X$ and $Y$, such that the commutation relation $[X, Y]=0$ be satisfied. This condition assures the existence of two coordinate parameters, namely $\tilde{t}=\tilde{t}(t, r)$ and $\tilde{r}=\tilde{r}(t, r)$, such that

$$
X=\frac{\partial}{\partial \tilde{t}}, Y=\frac{\partial}{\partial \tilde{r}}
$$

We decompose these fields

$$
\begin{aligned}
& Y=\lambda \bar{Y}, \quad \bar{Y}^{2}=1, \\
& X=a \bar{Y}+b \bar{Y}^{\perp}, \quad \bar{Y} \cdot \bar{Y}^{\perp}=0,
\end{aligned}
$$

with $b \neq 0$ and $\lambda>0$, and fixing equal to one the coefficient of $\frac{\partial}{\partial t}$ in the decomposition of $\bar{Y}^{\perp}$ in terms of $\frac{\partial}{\partial t}$ and $\frac{\partial}{\partial r}$. The condition $\bar{Y}^{2}=1$ is equivalent to

$$
\bar{Y}=f \frac{\partial}{\partial t}+P \frac{\partial}{\partial r}, \quad P=B^{-1}\left(-f C+\epsilon \sqrt{f^{2} l^{2}+B}\right)
$$

being $f$ an unknown function to be determined, $\epsilon= \pm 1$ and $l^{2}=-A B+C^{2}>0$. Moreover, $\bar{Y} \cdot \bar{Y}^{\perp}=0$ leads to

$$
\bar{Y}^{\perp}=\frac{\partial}{\partial t}+Q \frac{\partial}{\partial r}, \quad Q=B^{-1}\left(-C+\frac{\epsilon f l^{2}}{\sqrt{f^{2} l^{2}+B}}\right)
$$


Consequently, the resulting fields are

$$
\begin{aligned}
& X=(a f+b) \frac{\partial}{\partial t}+(a P+b Q) \frac{\partial}{\partial r}, \\
& Y=\lambda\left(f \frac{\partial}{\partial t}+P \frac{\partial}{\partial r}\right)=\lambda\left(f \alpha n+\frac{\epsilon}{B} \sqrt{f^{2} l^{2}+B} \frac{\partial}{\partial r}\right),
\end{aligned}
$$

where we have taken into account Eqs. (21) and (11). Using this decomposition, we will obtain an equation, involving only the unknown $f$, that gives a maximal family of hypersurfaces $\tilde{t}=$ constant. Notice that Eq. (14) makes clear the meaning of $\epsilon$ : relative to the Eulerian observer $n$, the radial component of $Y$ is outgoing or ingoing according to $\epsilon=1$ or $\epsilon=-1$.

The Jacobian matrix of the above change of coordinates can be written, in terms of the previous decompositions, as

$$
\begin{aligned}
\left(\begin{array}{cc}
\dot{\tilde{t}} & \tilde{t}^{\prime} \\
\dot{\tilde{r}} & \tilde{r}^{\prime}
\end{array}\right)= & \left(\begin{array}{ll}
\frac{\partial t}{\partial \tilde{t}} & \frac{\partial t}{\partial \tilde{r}} \\
\frac{\partial r}{\partial \tilde{t}} & \frac{\partial r}{\partial \tilde{r}}
\end{array}\right)^{-1}=\left(\begin{array}{cc}
a f+b & \lambda f \\
a P+b Q & \lambda P
\end{array}\right)^{-1} \\
& =(b p \lambda)^{-1}\left(\begin{array}{cc}
\lambda P & -\lambda f \\
-(a P+b Q) & (a f+b)
\end{array}\right),
\end{aligned}
$$

where $p=P-f Q=\frac{\epsilon}{\sqrt{f^{2} l^{2}+B}} \neq 0$. In order to ensure Eq. (8), we must require that $[X, Y]=0$, and this condition is expressed according to the following result.

Lemma 1: For the fields $X, Y$ given by Eqs. (13) and (14), the condition $[X, Y]=0$ is equivalent to

$$
\begin{array}{r}
{\left[\frac{P}{b p}\right]^{\prime}=\left[\frac{-f}{b p}\right]^{.},} \\
{\left[-\frac{a P+b Q}{b p \lambda}\right]^{\prime}=\left[\frac{a f+b}{b p \lambda}\right]^{.} .}
\end{array}
$$

To avoid confusion, we denote with $\widetilde{K}$ the trace of the extrinsic curvature of the new hypersurfaces $\tilde{t}=$ constant. From Eq. (4), the maximal slicing condition for the new hypersurfaces, $\widetilde{K}=0$, is equivalent to

$$
X\left(Y^{2}\right)+2 Y^{2} \frac{X(D)}{D}-2 Y(X \cdot Y)+\left[\frac{Y\left(Y^{2}\right)}{Y^{2}}-2 \frac{Y(D)}{D}\right] X \cdot Y=0 .
$$

Taking into account Eq. (18) and the commutation relation, we have 3 equations for 4 unknown functions, $a, b, f, \lambda$. But, due to $D$ transforms as a scalar under the considered 
coordinate transformation, we can add without loss of generality, the coordinate condition

$$
\tilde{r}^{2} Y^{2}=D
$$

saying that the metric on the hypersurfaces $\tilde{t}=$ constant is written in isotropic conformally flat form. Notice that Eq. (19) guarantees that the congruence of coordinate lines associated with the field $X=\frac{\partial}{\partial \tilde{t}}$ has minimal distortion. 9115

From Eq. (18), taking into account the conformally flat coordinate condition (19), we obtain the following result.

Lemma 2: The condition that the hypersurfaces $\tilde{t}=$ constant are maximal $(\widetilde{K}=0)$, is equivalent to

$$
2 Y(X \cdot Y)-3 X\left(Y^{2}\right)+\left[\frac{4}{\tilde{r}}+\frac{Y\left(Y^{2}\right)}{Y^{2}}\right] X \cdot Y=0,
$$

where $X, Y$ and $\tilde{r}$ are defined by Eqs. (19), (14) and (19).

Lemma 2 expresses the maximal slicing condition in terms of the fields $X, Y$. These fields have to satisfy the commutation relation given by Lemma 1. In the next section, we rewrite both conditions in terms of the decompositions (13) and (14).

\section{Local existence of maximal slicings}

In this section, we deduce the theorem stating the local existence of maximal slicings in SSST. The proof consists in reducing the required conditions into a set of first order partial differential equations.

In terms of the decompositions (13) and (14), Eqs. (19) and (20) are expressed as

$$
\begin{aligned}
& \lambda=\sqrt{D} / \tilde{r} \\
& f \dot{a}+P a^{\prime}-a\left(\frac{f \dot{\lambda}+P \lambda^{\prime}}{\lambda}-\frac{2}{\sqrt{D}}\right)=3 b \frac{\dot{\lambda}+Q \lambda^{\prime}}{\lambda} .
\end{aligned}
$$

Eq. (21) can be viewed as a definition of $\tilde{r}$ in terms of $\lambda$, and from this equation we can derive

$$
\frac{\lambda^{\prime}}{\lambda}=\frac{D^{\prime}}{2 D}-\frac{a f+b}{b p \sqrt{D}}, \quad \frac{\dot{\lambda}}{\lambda}=\frac{\dot{D}}{2 D}+\frac{a P+b Q}{b p \sqrt{D}},
$$

where we have taken into account the expression of the Jacobian matrix given by Eq. (15).

At this point we have 5 equations, i.e., Eqs. (16), (17), (22) and (23), for 4 unknown functions, i.e., $(a, b, f, \lambda)$. Eqs. (23) are equivalent to give quantity $a$ in terms of the other 
unknowns,

$$
a=b \sqrt{D}\left(\frac{\dot{\lambda}+Q \lambda^{\prime}}{\lambda}-\frac{\dot{D}+Q D^{\prime}}{2 D}\right),
$$

and to the following equation

$$
f \frac{\dot{\lambda}}{\lambda}+P \frac{\lambda^{\prime}}{\lambda}=f \frac{\dot{D}}{2 D}+P \frac{D^{\prime}}{2 D}-\frac{1}{\sqrt{D}} .
$$

Once $a$ has been eliminated, it can be seen that Eq. (17) can be obtained from Eq. (25). From Eq. (24), the field $X$ given by Eq. (13) is expressed as

$$
X=b p \sqrt{D}\left(-\Lambda^{\prime} \frac{\partial}{\partial t}+\dot{\Lambda} \frac{\partial}{\partial r}\right)
$$

where $\Lambda=\log \lambda / \sqrt{D}$. So we have reduced the problem to solve 3 equations, i.e., Eqs. (16), (22) (replacing $a$ ), and (25), for 3 unknown functions, i.e., $(b, f, \lambda)$. Eq. (16) can be rewritten as

$$
f \frac{\dot{b}}{b}+P \frac{b^{\prime}}{b}=P^{\prime}-P \frac{p^{\prime}}{p}+\dot{f}-f \frac{\dot{p}}{p} .
$$

As it will be shown below in Eq. (28), $f$ can be obtained from an equation which does not contain other variables. Then, Eq. (27) is a first order partial differential equation (PDE) for $b$.

Eliminating $a$ and its derivatives with Eq. (24), and making use of Eq. (27) to eliminate $b$, Eq. (22) is reduced to a second order PDE involving $\lambda$ and $f$. Finally, making use of Eq. (25) for, firstly, reducing the order of the equation and, secondly, for eliminating $\lambda$, Eq. (22) is equivalent to the following one:

$$
\frac{\dot{p}}{p}-\frac{\dot{D}}{D}-Q^{\prime}+Q\left[\frac{p^{\prime}}{p}-\frac{D^{\prime}}{D}\right]=0 .
$$

Notice that previous equation involves only $f$ when $p$ and $Q$ are written explicitly in terms of $f$ from their definitions.

We have found a decoupled system of quasi-linear PDEs (25), (27) and (28) according to the following steps: First, Eq. (28) can be solved for $f$. Second, Eq. (25) can be solved for $\lambda$. Third, Eq. (27) can be solved for $b$. Finally, $a$ can be obtained from Eq. (24). Assuming that all the metric components $A, B, C, D$ are continuously differentiable functions, the initial value problem with respect to this set of equations has always local (both

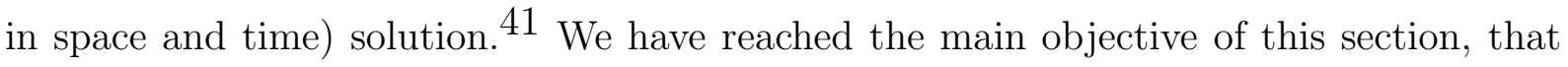
is to prove the following result. 
Theorem: Any spherically symmetric spacetime can be locally sliced by a family of maximal spacelike hypersurfaces.

Let us make a few comments about first order partial differential equations. If the functions appearing in the equation are continuously differentiable and we are looking for smooth solutions, given continuously differentiable initial data, it always exists a solution of the equation in a domain containing the given initial data. The domain of the solution can be the domain of initial parameters or a smaller one; so that is why, in principle, one can only guarantee local existence of solutions. Moreover, in this kind of equations one can use the so-called "characteristic method" in order to obtain, analytical or numerically, solutions of the equation. This method is based on two facts: i) given initial data at a point, it always exists a characteristic curve containing the given initial data; ii) a solution of the equation can be constructed by the union of a set of characteristic curves. So, a solution of the equation can be obtained by solving a set of characteristic curves around a given point.

Let us also point out that in the resolution of the characteristic curves some functional relations involving the dependent and independent variables have to be constant; the cardinal of the set of independent functional relations depends on the number of independent variables of the equation, and the general solution of the first order partial differential equation can be written as an implicit function of those ones.

Once the local existence of maximal slicings has been proved, we are going (see next Section) to design a practical procedure to build up these slicings.

\section{A procedure to construct maximal slicings}

The proof of the above theorem was based on the explicit finding of the minimum set of equations to be solved in order to obtain maximal slicings in SSSTs. Let us analyze a constructive algorithm that complements the theorem. We consider two different cases, $f=0$ and $f \neq 0$.

\subsection{First case $f=0$}

If $f=0$, Eq. (28) is reduced to $K=0$, the maximal slicing condition provided by Eq. (44). In this case, from Eqs. (27), (25) and (24), it turns out that $b(t) \neq 0$ arbitrary, 
$\lambda=Z(t) \sqrt{D} \exp \left(-\epsilon \int_{R}^{r} \sqrt{B / D} d r\right)$, with $Z(t)>0$ arbitrary and $R$ constant, and

$$
a=b \sqrt{D}\left[\dot{Z} / Z+\epsilon C / \sqrt{B D}-\epsilon \int_{R}^{r}(\sqrt{B / D}) \cdot d r\right] .
$$

The fields $X$ and $Y$ can be integrated according to:

Proposition 1: If the hypersurfaces $t=$ constant in the spacetime defined by the metric (11) are maximal, the following change of coordinates

$$
\tilde{t}=\tilde{t}(t), \quad \tilde{r}=Z^{-1} \exp \left(\epsilon \int_{R}^{r} \sqrt{B / D} d r\right),
$$

with $\dot{\tilde{t}}=b^{-1}, Z(t)>0$ and $R$ constant, allows one to write the metric in isotropic form

$$
\begin{aligned}
d s^{2}= & b^{2}\left[A+D\left(\frac{\dot{\tilde{r}}}{\tilde{r}}\right)^{2}-2 \epsilon C \sqrt{\frac{D}{B}} \frac{\dot{\tilde{r}}}{\tilde{r}}\right] d \tilde{t}^{2}+2 \frac{\epsilon b}{\tilde{r}} \sqrt{\frac{D}{B}}\left(C-\epsilon \sqrt{B D} \frac{\dot{\tilde{r}}}{\tilde{r}}\right) d \tilde{t} d \tilde{r} \\
& +\frac{D}{\tilde{r}^{2}}\left(d \tilde{r}^{2}+\tilde{r}^{2} d \Omega^{2}\right) .
\end{aligned}
$$

This metric form is adapted to the same initial maximal slices, that are now labeled as $\tilde{t}=$ constant. It remains invariant under the inversion $\tilde{r} \rightarrow T(\tilde{t}) / \tilde{r}$ when, in addition, the sign of the radial component of the field $Y$ is reversed by the change $\epsilon \rightarrow-\epsilon$.

\subsection{Second case $f \neq 0$}

When $f \neq 0$, the explicit expressions of $P / f$ and $Q$ in terms of $f$, allow us to define the variable

$$
F=\frac{\epsilon f}{\sqrt{f^{2} l^{2}+B}} \quad \Leftrightarrow \quad f=\epsilon F \sqrt{\frac{B}{1-l^{2} F^{2}}} .
$$

Then, using the definition of $P$ and $Q$ given by Eqs. (11) and (12) respectively, we have:

$$
\frac{P}{f}=\frac{1-F C}{F B}, \quad Q=\frac{-C+F l^{2}}{B}, \quad p=\epsilon \sqrt{\frac{1-l^{2} F^{2}}{B}} .
$$


Eqs. (27), (25) and (28) are rewritten, respectively, as

$$
\begin{aligned}
\dot{b}+\frac{1-F C}{F B} b^{\prime}= & b\left\{\frac{\dot{B}-C^{\prime}}{B}+\frac{1}{1-l^{2} F^{2}}\left[-\left(1+l^{2} F^{2}\right) \frac{\dot{F}}{F}+\frac{l^{2} F(2-F C)-C}{B} \frac{F^{\prime}}{F}\right.\right. \\
& \left.\left.+F^{2}\left(l^{2}\right)^{\cdot}+\frac{(1-F C) F\left(l^{2}\right)^{\prime}}{B}\right]\right\} \\
\dot{\lambda}+\frac{1-F C}{F B} \lambda^{\prime}= & \lambda\left[\frac{\dot{D}}{2 D}+\frac{1-F C}{F B} \frac{D^{\prime}}{2 D}-\frac{\epsilon \sqrt{1-l^{2} F^{2}}}{F \sqrt{B D}}\right], \\
\dot{F}+\frac{1-F C}{F B} F^{\prime}= & -\frac{F B\left(l^{2}\right)^{\cdot}+\left(2-l^{2} F^{2}-F C\right)\left(l^{2}\right)^{\prime}}{2 l^{2} B}-\left[\frac{\dot{B}}{2}+\frac{B \dot{D}}{D}+\frac{\left(C-F l^{2}\right)\left(D B^{\prime}-2 B D^{\prime}\right)}{2 B D}-C^{\prime}\right] \\
- & L
\end{aligned}
$$

where $L=\left(1-l^{2} F^{2}\right) /\left(l^{2} F B\right)$. The solution can be obtained numerically solving the quasilinear Eqs. (35), (34) and (33) for, respectively, $F, \lambda$ and $b$, given initial data for $t=t_{0}$. These equations are of hyperbolic type, analogous to the well-known advective equation in classical fluid dynamics. The three equations have a common "advective velocity": $V=(1-F C) /(F B)$. This velocity can be interpreted as the radial component of the light velocity in the modified metric $d \tilde{s}^{2}=d s^{2}-\frac{1}{f^{2}} d t^{2}$.

On the other hand, the coordinate 1 -forms $d \tilde{t}$ and $d \tilde{r}$ are easily written from Eq. (15), taking into account Eqs. (32). Then, we arrive to this result:

Proposition 2: For any given SSST a maximal slicing can be built up according to the following steps:

(i) To start with the metric form (11), with $l^{2} \equiv C^{2}-A B>0, B>0$ and $D>0$, to solve Eq. (35) for $F$, and then, to solve Eq. (33) for $b$.

(ii) To define

$$
\Gamma \equiv b^{-1}\left(1-l^{2} F^{2}\right)^{-1}[(1-F C) d t-F B d r]
$$

which is necessarily a closed 1-form.

(iii) To find a potential $\tilde{t}(t, r)$ of $\Gamma, \Gamma=d \tilde{t}$. Then, the level surfaces of $\tilde{t}$ are spacelike and maximal.

Analogously, by applying Eq. (21), $\tilde{r}$ can be integrated once $F$ and $\lambda$ are solved. The change of coordinates $\{\tilde{t}=\tilde{t}(t, r), \tilde{r}=\tilde{r}(t, r), \theta, \varphi\}$ is completely defined from the fields 
$X, Y$ given by Eqs. (13) and (14). Then, the metric form can be deduced in the new set of coordinates. The new slices are maximal and Proposition 1 can again be applied.

\section{Maximal slicings in flat spacetime}

In order to illustrate how the procedure designed in previous section works, let us consider the simple case of the Minkowski spacetime starting with inertial spherical coordinates, $d s^{2}=-d t^{2}+d r^{2}+r^{2} d \Omega^{2}$. According to Proposition $1, \tilde{t}=\tilde{t}(t)$, with $\dot{\tilde{t}}=b^{-1}, \tilde{r}=r^{\epsilon} Z^{-1}$, and the resulting metric is

$$
d s^{2}=\left[-b^{2}+\left(\tilde{r}^{\epsilon} Z^{\epsilon-1} \frac{d Z}{d \tilde{t}}\right)^{2}\right] d \tilde{t}^{2}+2(\tilde{r} Z)^{2 \epsilon-1} \frac{d Z}{d \tilde{t}} d \tilde{t} d \tilde{r}+\left(\tilde{r}^{\epsilon-1} Z^{\epsilon}\right)^{2}\left(d \tilde{r}^{2}+\tilde{r}^{2} d \Omega^{2}\right)
$$

$b=b(\tilde{t}) \neq 0, Z=Z(\tilde{t})>0$ being arbitrary functions. So, we have the same slicing that the initial inertial one (this foliation is totally geodesic, i.e., its extrinsic curvature tensor vanishes). However, other maximal slicings (non-inertial, in general) can be obtained as a consequence of Proposition 2. According to it, Eq. (35) reads

$$
\dot{F}+F^{-1} F^{\prime}=-2 r^{-1}\left(1-F^{2}\right) .
$$

Following the last comments of Sec. 4, the general solution of this equation can be given in terms of an implicit function,

$$
\Phi_{1}\left(m(F)+t r^{-1}\left(F^{-2}-1\right)^{1 / 4}, r^{-1}\left(F^{-2}-1\right)^{1 / 4}\right)=0
$$

with $0<F^{2}<1, m(F)=4 \sqrt{|F|}{ }_{2} \mathrm{~F}_{1}\left(1 / 4,3 / 4 ; 5 / 4 ; F^{2}\right),{ }_{2} \mathrm{~F}_{1}$ being the classical standard hypergeometric series 42 and $\Phi_{1}$ being an arbitrary function. Once $F$ is known, the general solution of Eqs. (33) and (34) are, respectively, $\Phi_{2}\left(I_{1}, I_{2}\right)$ and $\Phi_{3}\left(I_{1}, I_{3}\right)$, where $\Phi_{2}$ and $\Phi_{3}$ are arbitrary functions, and

$$
\begin{aligned}
& I_{1}=r-\int_{0}^{t} F^{-1} d t \\
& I_{2}=b^{-1} \exp \left[\int_{0}^{t}\left(-\frac{\left(1+F^{2}\right) \dot{F}}{F}+2 F^{\prime}\right) \frac{d t}{1-F^{2}}\right] \\
& I_{3}=\lambda^{-1} \exp \left(r^{-1} \int_{0}^{t} F^{-1}\left(1-\epsilon \sqrt{1-F^{2}}\right) d t\right) .
\end{aligned}
$$


This study can be extended to the Schwarzschild spacetime using different metric forms, specially those that are regular at the horizon, like the Painlevé-Gullstrand form and other similar ones. $\frac{43}{4}$ Maximal slincings can also be constructed in other spacetimes (ReissnerNordtröm, Vaidya, Lemaître-Tolman-Bondi, etc.) and the results compared with previous works. $20|21| 22|23| 24$

\section{Maximal slicings in closed Friedmann spacetime}

The pioneering analysis by Oppenheimer and Snyder $\underline{34}$ on the dynamics of the collapse of a homogeneous sphere of pressure-zero matter allowed them to obtain an analytical solution of Einstein equations for a dynamical spacetime. Its metric, a special case of LemaîtreTolman-Bondi metric, has a simple form (see below), being the Friedmann metric in the interior. We are going to apply the procedure designed in Sec. 5 to build maximal slicings in a closed Friedmann spacetime. We start with the following metric form:

$$
d s^{2}=R^{2}\left(-d \eta^{2}+d \chi^{2}+\sin ^{2} \chi d \Omega^{2}\right),
$$

where $R=R_{0}(1-\cos \eta), R_{0}$ being a positive constant, and $\eta \in(-\pi, 0), \chi \in(0, \pi)$, being the domain of the parameters ( $\eta$ and $\chi$ will play the role of $t$ and $r$ in Eq. (11) respectively). The hypersurfaces $\eta=$ constant are not maximal. We are going to solve numerically Eqs. (28), (27) and (25). Let us define $\tilde{f}:=R f$.

Eq. (28) reads

$$
\frac{\tilde{f}}{1+\tilde{f}^{2}} \frac{\partial \tilde{f}}{\partial \eta}+\frac{\epsilon}{\sqrt{1+\tilde{f}^{2}}} \frac{\partial \tilde{f}}{\partial \chi}+6 \cot \frac{\eta}{2}+\frac{2 \epsilon \tilde{f} \cot \chi}{\sqrt{1+\tilde{f}^{2}}}=0
$$

Without loss of generality, it can be considered $\epsilon=1$, since for $\epsilon=-1$ the solution will be $-\tilde{f}$. Previous equation is then equivalent to

$$
\frac{\tilde{f}}{\sqrt{1+\tilde{f}^{2}}} \frac{\partial \tilde{f}}{\partial \eta}+\frac{\partial \tilde{f}}{\partial \chi}=-6 \sqrt{1+\tilde{f}^{2}} \cot \frac{\eta}{2}-2 \tilde{f} \cot \chi
$$

We use the characteristic method for solving the above equation (44). Let $(\eta(\mu), \chi(\mu), \tilde{f}(\mu))$ be a characteristic curve depending on a parameter $\mu$ such that $\frac{d \eta}{d \mu}=\frac{\tilde{f}}{\sqrt{1+\tilde{f}^{2}}}, \frac{d \chi}{d \mu}=1$, and $\frac{d \tilde{f}}{d \mu}=-6 \sqrt{1+\tilde{f}^{2}} \cot \frac{\eta}{2}-2 \tilde{f} \cot \chi$. Substituting $d \chi=d \mu$, and considering then 


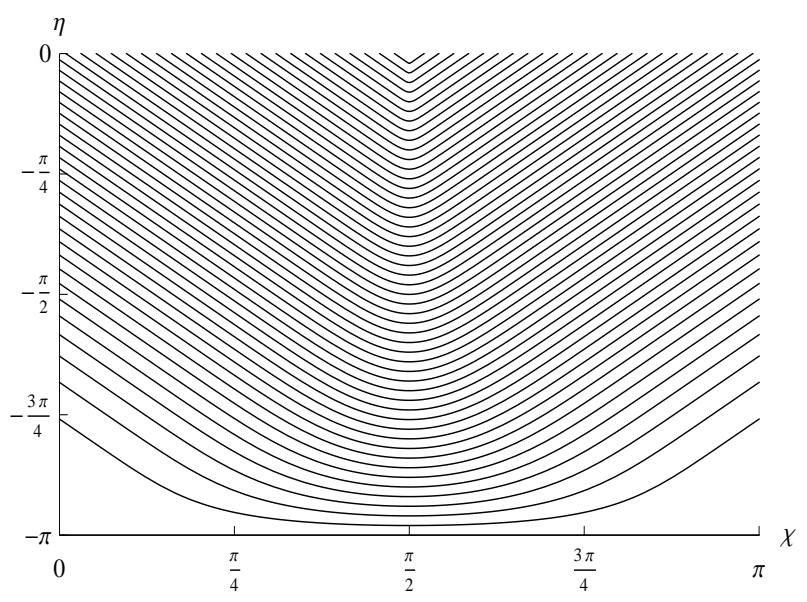

Figure 1: Projection onto the $\eta-\chi$ plane of characteristic curves of Eqs. (45), with $\tilde{f}(\chi=\pi / 2)=0, \eta(\chi=\pi / 2)=-k \pi, 0<k \leq 1$, initial data.

$(\eta(\chi), \tilde{f}(\chi))$, the characteristic system associated to Eq. (44) is written as

$$
d \eta / d \chi=\tilde{f} / \sqrt{1+\tilde{f}^{2}}, d \tilde{f} / d \chi=-6 \sqrt{1+\tilde{f}^{2}} \cot \frac{\eta}{2}-2 \tilde{f} \cot \chi .
$$

Notice that, although $\eta=-\pi$ is out of the range of the parameter, $\tilde{f}=0, \eta=-\pi$ is a solution of the previous system. Notice also that solutions of Eqs. (45) satisfy $\tilde{f}\left(\frac{\pi}{2}-x\right)=$ $-\tilde{f}\left(\frac{\pi}{2}+x\right)$ and $\eta\left(\frac{\pi}{2}-x\right)=\eta\left(\frac{\pi}{2}+x\right)$. Eqs. (45) can be solved numerically, imposing

$$
\tilde{f}(\chi=\pi / 2)=0, \eta(\chi=\pi / 2)=-k \pi, 0<k \leq 1,
$$

as initial data. In Fig. 1 we plot the projection onto the $\eta-\chi$ plane of the characteristic curves, for the values $k=-l / 50, l=1, \ldots, 50$. In Fig. 2 we plot the projection onto the $\tilde{f}-\chi$ plane of the characteristic curves, for the values $k=l / 5, l=1, \ldots, 4$. The union of the characteristic curves, for all the possible values of $k, 0<k \leq 1$, covers the whole domain of parameters for $\eta$ and $\chi$. This union constitutes a global solution of Eq. (44) for $\tilde{f}$.

Once $\tilde{f}$ (and therefore $f$ ) is solved, we focus on Eq. (27) for $b$, which reads (after multiplying by $\epsilon R$ )

$$
\epsilon \tilde{f} \frac{\partial \log b}{\partial \eta}+\sqrt{1+\tilde{f}^{2}} \frac{\partial \log b}{\partial \chi}=\frac{\partial \tilde{f}}{\partial \eta}\left[\frac{\tilde{f}}{\sqrt{1+\tilde{f}^{2}}}+\epsilon\left(1+\frac{\tilde{f}^{2}}{1+\tilde{f}^{2}}\right)\right]+\frac{\tilde{f}}{\sqrt{1+\tilde{f}^{2}}} \frac{\partial \tilde{f}}{\partial \chi}
$$

From Eq. (44), the combinations $\epsilon \tilde{f}$ and $\tilde{f}^{2}$ will remain the same for both values of $\epsilon$. Without loss of generality, we take $\epsilon=1$ and the previous solution for $\tilde{f}$. Due to 


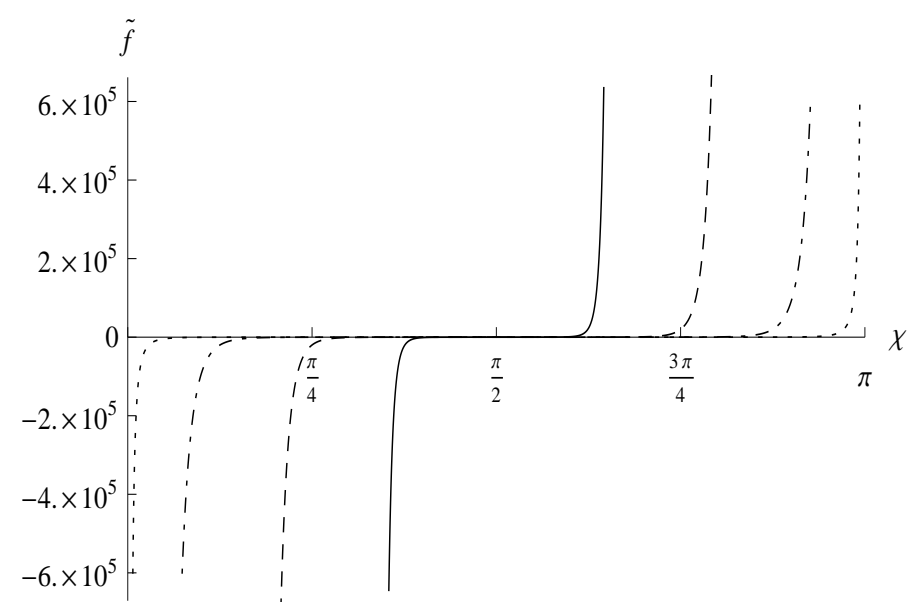

Figure 2: Projection onto the $\tilde{f}-\chi$ plane of characteristic curves of Eqs. (45), with $\tilde{f}(\chi=\pi / 2)=0, \eta(\chi=\pi / 2)=-k \pi, k=1 / 5$ (solid line), $2 / 5$ (dashed line), $3 / 5$ (dash-dotted line), 4/5 (dotted line) initial data.

numerical reasons, we substitute (using Eq. (44) $) \frac{\partial \tilde{f}}{\partial \chi}$ in terms of $\frac{\partial \tilde{f}}{\partial \eta}$, leading to

$$
\begin{aligned}
\frac{\tilde{f}}{\sqrt{1+\tilde{f}^{2}}} \frac{\partial \log b}{\partial \eta}+\frac{\partial \log b}{\partial \chi}= & \frac{\partial \tilde{f} / \partial \eta}{\sqrt{1+\tilde{f}^{2}}}\left(\frac{\tilde{f}}{\sqrt{1+\tilde{f}^{2}}}+1\right) \\
& -\frac{2 \tilde{f}^{2}}{1+\tilde{f}^{2}} \cot \chi-\frac{6 \tilde{f}}{\sqrt{1+\tilde{f}^{2}}} \cot \frac{\eta}{2}
\end{aligned}
$$

We use again the characteristic method for solving the above equation (48). Let us consider a characteristic curve $(\eta(\chi), \log b(\chi))$, such that

$$
\begin{aligned}
& d \eta / d \chi=\tilde{f} / \sqrt{1+\tilde{f}^{2}} \\
& \frac{d \log b}{d \chi}=\frac{\partial \tilde{f} / \partial \eta}{\sqrt{1+\tilde{f}^{2}}}\left(\frac{\tilde{f}}{\sqrt{1+\tilde{f}^{2}}}+1\right)-\frac{2 \tilde{f}^{2}}{1+\tilde{f}^{2}} \cot \chi-\frac{6 \tilde{f}}{\sqrt{1+\tilde{f}^{2}}} \cot \frac{\eta}{2}
\end{aligned}
$$

Notice that the projection onto the $\eta-\chi$ plane will be exactly the same as the one for the characteristic curves associated to $\tilde{f}$. In practice, the characteristic curves for $\log b$ can be calculated simultaneously to the ones for $\tilde{f}$. Notice also that, although $\eta=-\pi$ is out of the range of the parameter, $b=1, \eta=-\pi$ (with $\tilde{f}=0$ ) is a solution of the previous system. Eqs. (49) can be solved numerically, imposing

$$
\log b(\chi=\pi / 2)=0
$$

and the previous values for $\tilde{f}$ and $\eta$ as initial data. In Fig. 3 we plot the projection onto the $\log b-\chi$ plane of the characteristic curves, for the values $k=l / 5, l=1, \ldots, 4$. The 


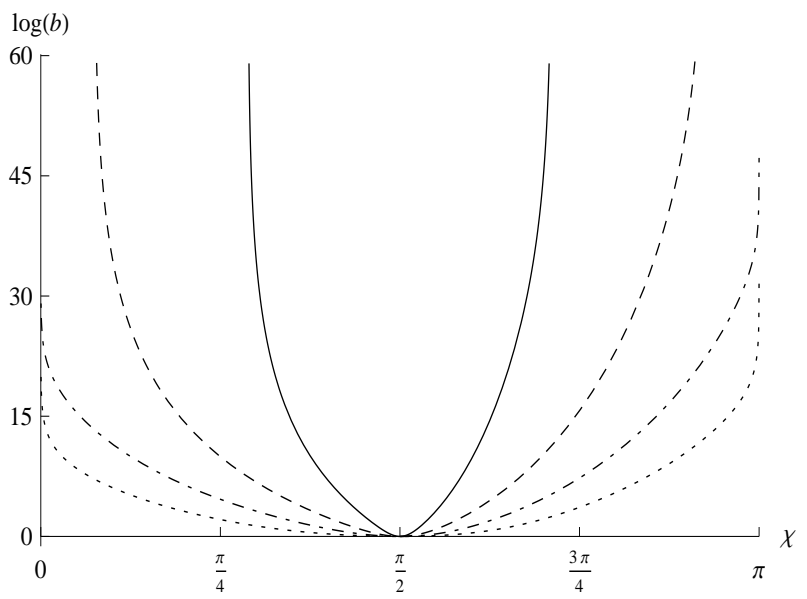

Figure 3: Projection onto the $\log b-\chi$ plane of characteristic curves of Eqs. (49), with $\log b(\chi=\pi / 2)=0$, previous values for $\tilde{f}$ and $\eta, k=1 / 5$ (solid line), 2/5 (dashed line), 3/5 (dash-dotted line), 4/5 (dotted line), initial data.

union of the characteristic curves, for all the possible values of $k, 0<k \leq 1$, covers the whole domain of parameters for $\eta$ and $\chi$. This union constitutes a global solution of Eq. (48) for $\log b . b \neq 0$ is satisfied.

We focus now on (25) for $\lambda$, which reads (after multiplying by $\epsilon R / \sqrt{1+\tilde{f}^{2}}$ )

$$
\frac{\epsilon \tilde{f}}{\sqrt{1+\tilde{f}^{2}}} \frac{\partial \log \lambda}{\partial \eta}+\frac{\partial \log \lambda}{\partial \chi}=\frac{2 \epsilon \tilde{f}}{\sqrt{1+\tilde{f}^{2}}} \cot \frac{\eta}{2}+\frac{\cos \chi-\epsilon / \sqrt{1+\tilde{f}^{2}}}{\sin \chi} .
$$

The combinations $\epsilon \tilde{f}$ and $\tilde{f}^{2}$ will remain the same for both values of $\epsilon$, so we can use previous solution for $\tilde{f}$ but we have to keep $\epsilon$ in the last term,

$$
\frac{\tilde{f}}{\sqrt{1+\tilde{f}^{2}}} \frac{\partial \log \lambda}{\partial \eta}+\frac{\partial \log \lambda}{\partial \chi}=\frac{2 \tilde{f}}{\sqrt{1+\tilde{f}^{2}}} \cot \frac{\eta}{2}+\frac{\cos \chi-\epsilon / \sqrt{1+\tilde{f}^{2}}}{\sin \chi} .
$$

Therefore, we will have two solutions $\log \lambda_{\epsilon}$ which do not differ only in the sign. Using again the characteristic method for solving the above equation (52), we consider a characteristic curve $(\eta(\chi), \log \lambda(\chi))$, such that

$$
d \eta / d \chi=\tilde{f} / \sqrt{1+\tilde{f}^{2}}, \quad \frac{d \log \lambda}{d \chi}=\frac{2 \tilde{f}}{\sqrt{1+\tilde{f}^{2}}} \cot \frac{\eta}{2}+\frac{\cos \chi-\epsilon / \sqrt{1+\tilde{f}^{2}}}{\sin \chi}
$$

The projection onto the $\eta-\chi$ plane is exactly the same as the one for the other characteristic curves. Hence, the corresponding projections for $\log \lambda$ can be calculated simultaneously to them. Notice that, although $\eta=-\pi$ is out of the range of the parameter, 


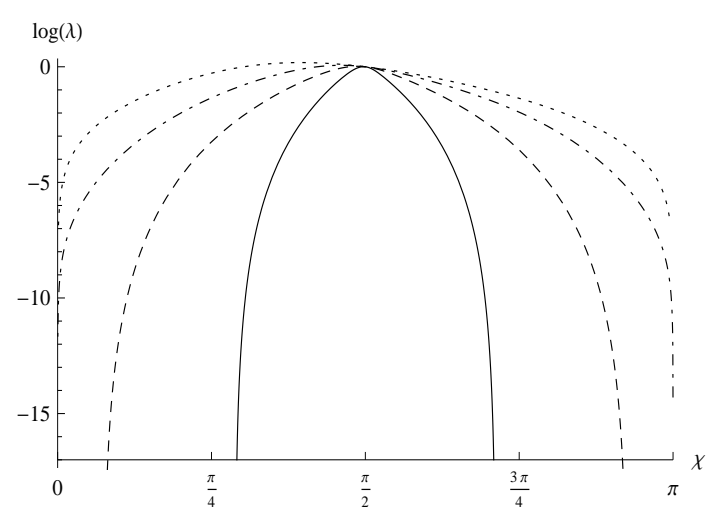

(a) $\epsilon=1$.

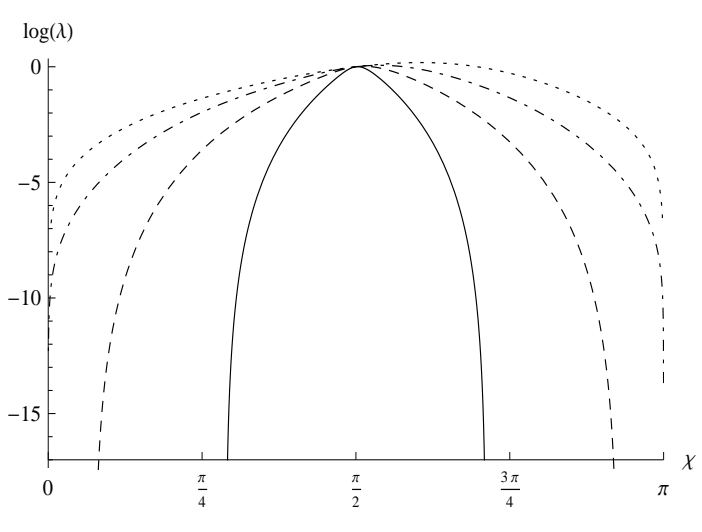

(b) $\epsilon=-1$.

Figure 4: Projection onto the $\log \lambda-\chi$ plane of characteristic curves of Eqs. (53), with $\log \lambda(\chi=\pi / 2)=0$, previous values for $\tilde{f}$ and $\eta, k=1 / 5$ (solid line), $2 / 5$ (dashed line), $3 / 5$ (dash-dotted line), 4/5 (dotted line), initial data.

$\lambda_{\epsilon}=\lambda_{0} \sin \chi(\cot \chi / 2)^{\epsilon}, \eta=-\pi, \lambda_{0}$ being a positive constant, is a solution of the previous system (with $\tilde{f}=0$ ). Eqs. (53) can be solved numerically, imposing

$$
\log \lambda(\chi=\pi / 2)=0
$$

and the previous values for $\tilde{f}$ and $\eta$ as initial data. These initial data corresponds to the value $\lambda_{0}=1$ in the limit $\eta=-\pi$. In Fig. 4 we plot the projection onto the $\log \lambda-\chi$ plane of the characteristic curves, for the values $k=l / 5, l=1, \ldots, 4$, and for both values of $\epsilon$. The union of the characteristic curves, for all the possible values of $k, 0<k \leq 1$, covers the whole domain of parameters for $\eta$ and $\chi$. This union constitutes a global solution of Eq. (152) for $\log \lambda . \lambda>0$ is satisfied.

Notice that the fact that the projection onto the $\eta-\chi$ plane of the characteristic curves for $\tilde{f}, \log b$ and $\log \lambda$ coincides is just a consequence of the fact that Eqs. (33)-(35) share the same advective velocity.

Once $\tilde{f}, b$ and $\lambda$ have been derived, we can calculate the unknown $a$ from Eq. (24),

$$
a=\frac{b R}{1+\tilde{f}^{2}}[\sin \chi(\partial \log \lambda / \partial \eta-2 \cot \eta / 2)-\tilde{f}] .
$$

Also, from Eq. (21), we can directly obtain

$$
\tilde{r}=\frac{R}{\lambda} \sin \chi
$$

In Fig. 5 we plot the projection onto the $\tilde{r}-\chi$ plane of the characteristic curves, for the values $k=l / 5, l=1, \ldots, 4$, and for both values of $\epsilon$. 

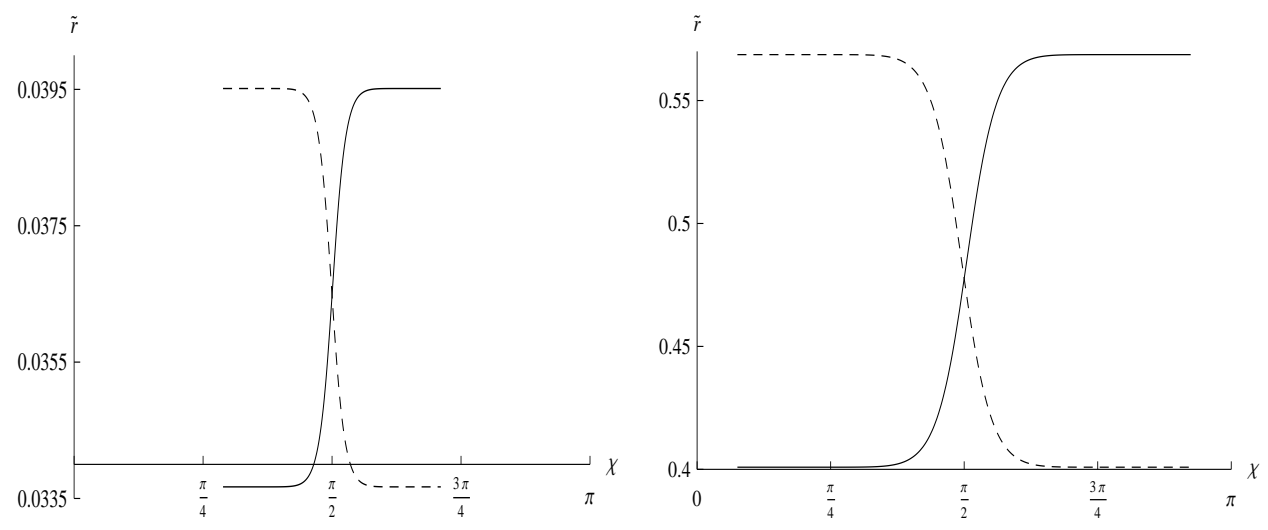

(a) $k=1 / 5$.

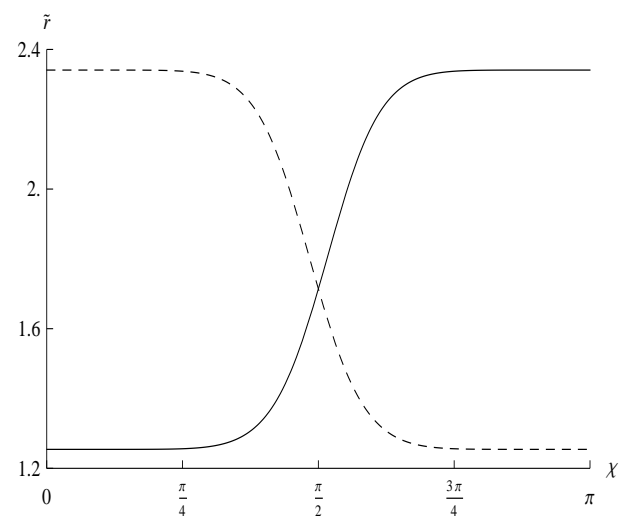

(b) $k=2 / 5$.

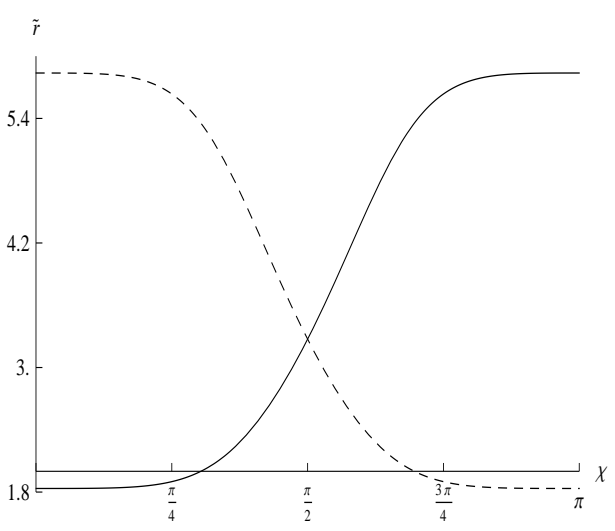

(c) $k=3 / 5$.

(d) $k=4 / 5$.

Figure 5: Projection of characteristic curves onto $\tilde{r}-\chi$ plane. Solid lines correspond to $\epsilon=1$, while dashed lines correspond to $\epsilon=-1$. 


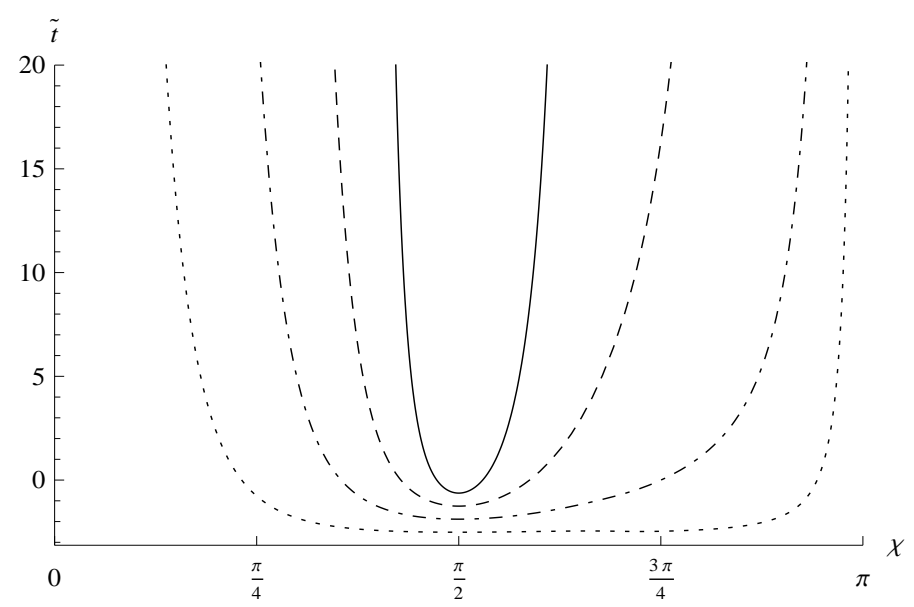

Figure 6: Projection onto the $\tilde{t}-\chi$ plane of characteristic curves, with $\tilde{t}(\chi=\pi / 2)=$ $\eta, \eta(\chi=\pi / 2)=-k \pi, k=1 / 5$ (solid line), 2/5 (dashed line), 3/5 (dash-dotted line), 4/5 (dotted line) initial data.

The conditions for the variable $\tilde{t}$ are the ones in Proposition 2, once $F$ is written in terms of $f$ and the components of the initial metric (42) in this particular case. From Eq. (36) (let us draw reader's attention to the fact that we are using $\eta$ and $\chi$ instead of $t$ and $r$, respectively), and the corresponding values of $\tilde{f}$ and $b, \tilde{t}$ has to satisfy $\partial \tilde{t} /\left.\partial \chi\right|_{\eta=-\pi}=$ 0, i.e., $\left.\tilde{t}\right|_{\eta=-\pi}$ has to be constant. Moreover, $\partial \tilde{t} /\left.\partial \eta\right|_{\chi=\pi / 2}=1$. We can choose consistently $\left.\tilde{t}\right|_{\chi=\pi / 2}=\eta$ and $\left.\tilde{t}\right|_{\eta=-\pi}=-\pi$. The values of $\tilde{t}$ in the whole domain of parameters can be numerically obtained from the expression of $\partial \tilde{t} / \partial \eta$, coefficient of $d \eta$ in Eq. (36) for $\Gamma$, and the condition $\left.\tilde{t}\right|_{\chi=\pi / 2}=\eta$. In Fig. 6 we plot the projection onto the $\tilde{t}-\chi$ plane of the characteristic curves, for the values $\tilde{t}(\chi=\pi / 2)=\eta, \eta(\chi=\pi / 2)=-k \pi, k=l / 5$, $l=1, \ldots, 4$.

Numerically, the metric (42) can be written in the new set of coordinates $\{\tilde{t}, \tilde{r}, \theta, \varphi\}$, evaluating the corresponding Jacobian matrix from (15). The hypersurfaces $\tilde{t}=$ constant are now maximal.

\section{Conclusions}

We have proved that any SSST can be locally sliced by a family of maximal spacelike hypersurfaces.

We have designed a general geometrical method to build up maximal slices in any SSST by solving three decoupled first order quasi-linear PDEs (25), (27) and (28). It has 
been applied in the cases of Minkowski (vacuum) and Friedmann (matter) spacetimes. In the first case, a flat spacetime, the equations leading to obtain maximal slicings, which are not inertial in general, can be solved analytically. In the second case, closed Friedmann spacetime, the corresponding equations leading to obtain maximal slicings are solved numerically.

An interesting by-product for Numerical Relativity of the approach presented in this paper has to do with the assessment of 3D codes written, as customary, in Cartesian coordinates. Let us consider two codes $\mathrm{NC} 1$ and $\mathrm{NC} 2$ such that only $\mathrm{NC} 1$ uses a gauge which is maximal. Hence, the evolution with code $\mathrm{NC} 2$ of any initial data admitting a spherically symmetric limit could be compared to the evolution produced by code NC1, by simply using our procedure to generate a SSST satisfying the maximal slicing condition. To emphasize this point we have applied our procedure to build up, numerically, maximal slicings in a Friedmann spacetime. If one takes into account that Oppenheimer-Snyder collapse is one of the basic test-beds for any numerical code aiming to solve Einstein equations, our analysis paves the way to calibrate numerical codes that use formalisms that do not incorporate the maximal slicing condition.

We have outlined a method that should be extended to deal with the construction of constant mean curvature slicings. The results on maximal slicings we have presented here can be considered as a step to gain some insights in this direction.

Let us make a comment about maximal hypersurfaces in a cosmological scenario. In the seventies, Brill, 44 and Brill and Flaherty 45 analyzed the existence and uniqueness of maximal hypersurfaces in a cosmological context. Brill concludes that closed surface of stationary area $(M S)$ in closed universes are exceptional and scarce $\frac{44}{4 n}$ particular, Brill finds: Any closed MS in a closed universe which satisfies Einstein's equations with the energy condition is a local maximal surface and is locally isolated. ${ }^{44}$ These authors reached these conclusions in analyzing non-static Friedmannian cosmologies, for which such a hypersurfaces are identified with instants of cosmic time. In fact, in these cosmologies, the volume functional (relative to the matter flow) might take a critical value only if the expansion of the cosmological observers vanishes, i.e. at a particular cosmological moment, depending on the particular closed universe. The above conclusions by Brill and Flaherty and the one presented in this paper, i.e., the local existence of a maximal slicing in any SSST, are compatible. In fact, the result by Bill and Flaherty involves the hypothesis of 
global spatial compacteness meanwhile our result concerns only local existence, and no global topological property has been required.

As we have pointed out from the very beginning, constructing maximal slicing are closely related to find a vorticity-free observer without expansion. Such an Eulerian observer is not necessary to be geodesic or shear-free, i.e., it is not (generically) a cosmological observer moving with the matter content of the universe. Then, as a corollary of the results we have presented, maximal slicings exist locally (and can be constructed) in any Robertson-Walker spacetime: they are those slicings which are associated with Eulerian observers without expansion.

Our statement about the local existence of a maximal slicing in any SSST, and the examples of constructions we have considered, support the physical interest in considering such a local foliations when their global existence is forbidden from topological requirements.

\section{Acknowledgements}

I. C.-C. acknowledges support from the Alexander von Humboldt Foundation. We wish to thank Dr. José Luis Jaramillo for detailed appreciations and interesting comments about this paper and related issues. This work has been also supported by the MICINN Grant No. AYA2007-67626-C03-01 and the MICINN-FEDER project No. FIS2009-07705.

\section{References}

1 A. Lichnerowicz, "L'integration des equations de la gravitation relativiste et le probleme des $N$ corps" J. Math. Pure Appl. 23 37-63 (1944).

2 Y. Choquet-Bruhat, "Maximal submanifolds and submanifolds with constant mean extrinsic curvature of a lorentzian manifold," Annali della Scuola Normale Superiore di Pisa Classe di Scienze $4^{e}$ sèrie, tome 3, number 3, 361-376 (1976).

3 M. Cantor, A. Fisher, J. Marsden, N. Ō Murchadha, and J. M. York, "The existence of Maximal Slicings in Asyptotically Flat Spacetimes," Commun. Math. Phys. 49, 187-190 (1976).

4 J. E. Marsden, and F. J. Tipler "Maximal hypersurfaces and foliations of constant 
mean curvature in general relativity," Phys. Rep. 66, 109-139 (1980).

5 R. Bartnik, "Existence of maximal surfaces in asymptotically flat spacetimes," Commun. Math. Phys. 94, 155-175 (1984).

${ }^{6}$ P. T. Chruściel, and R. W. Wald, "Maximal hypersurfaces in stationary asymptotically flat spacetimes," Commun. Math. Phys. 163, 561-604 (1994).

7 G. A. Burnett, and A. L. Rendall, "Existence of maximal hypersurfaces in some spherically symmetric spacetimes," Classical and Quantum Gravity, 13, 111-123 (1996).

8 Y. Choquet-Bruhat, General Relativity and the Einstein Equations (Oxford University Press, Oxford, 2008).

${ }^{9}$ L. Smarr, and J. W. York, "Kinematical conditions in the construction of spacetime," Phys. Rev. D 17, 2529-2551 (1978).

10 L. Smarr, and J. W. York, "Radiation gauge in general relativity," Phys. Rev. D 17, 1945-1956 (1978).

11 M. Shibata, and T. Nakamura, "Evolution of three-dimensional gravitational waves: Harmonic slicing case," Phys. Rev. D 52, 5428-5444 (1995).

12 J. A. Isenberg, "Waveless Approximation Theories of Gravity," Int. J. Mod. Phys. D 17, 265-273 (2008).

13 S. Bonazzola, E. Gourgoulhon, P. Grandclément, and J. Novak, "Constrained scheme for the Einstein equations based on the Dirac gauge and spherical coordinates," Phys. Rev. D, 70104007 (2004).

14 L-M. Lin, and J. Novak, "Rotating star initial data for a constrained scheme in numerical relativity," Classical and Quantum Gravity 23, 4545-4561 (2006).

15 E. Gourgoulhon, 3+1 Formalism and bases of Numerical Relativity, Lectures at Institute Henri Poincaré (Paris, 2006), arXiv:gr-qc/0703035.

16 M. Alcubierre, Introduction to 3+1 Numerical Relativity (Oxford University Press, Oxford, 2008).

17 R. Schoen, and S. T. Yau, "On the proof of the positive mass conjecture in general relativity," Commun. Math. Phys. 65, 45-76 (1979).

18 R. Schoen, and S. T. Yau, "Proof of the positive mass theorem. II." Commun. Math. Phys. 79, 231-260 (1981).

19 J. W. York, "Kinematics and dynamics of General relativity," Sources of Gravita- 
tional Radiation, Edited by L. L. Smarr, (Cambridge University Press, Cambridge 1979) pp. 83-126.

20 F. Estabrook, H. Wahlquist, S. Christensen, B. DeWitt, L. Smarr, and E. Tsiang, "Maximally Slicing a Black Hole," Phys. Rev. D 7, 2814-2817 (1973).

21 R. Beig, and N. Ó Murchadha, "Late time behavior of the maximal slicing of the Schwarzschild black hole," Phys. Rev. D 57, 4728-4737 (1998).

22 B. L. Reinhart, "Maximal foliations of extended Schwarzschild space," J. Math. Phys. 14, 719 (1973).

23 E. Malec, "Event horizons and apparent horizons in spherically symmetric geometries," Phys. Rev. D 49, 6475-6483 (1994).

24 L. I. Petrich, S. L. Shapiro, and S. A. Teukolsky, "Oppenheimer-Snyder collapse with maximal time slicing and isotropic coordinates," Phys. Rev. D 31, 2459-2469 (1985).

25 D. M. Eardley, and L. Smarr, "Time functions in numerical relativity: Marginally bound dust collapse," Phys. Rev. D 19, 2239-2259 (1979).

26 J. Guven, and N. Ó Murchadha, "Flat foliations of spherically symmetric geometries," Phys. Rev. D 60, 104015 (1999).

27 V. Husain, A. Qadir, and A. A. Siddiqui, "Note on flat foliations of spherically symmetric spacetimes," Phys. Rev. D 65, 027501 (2001).

28 R. Beig, and A. A. Siddiqui, "Uniqueness of flat spherically symmetric spacelike hypersurfaces admitted by spherically symmetric static spacetimes," Classical and Quantum Gravity 24, 5435-5439 (2007).

29 C. Doran, "New form of the Kerr solution," Phys. Rev. D 61, 067503 (2000).

30 J. Natário, "Painlevé-Gullstrand coordinates for the Kerr solution," Gen. Relativ. Gravit. 41, 2579-2586 (2009).

31 C-Y. Lin and C Soo, "Generalized Painlevé-Gullstrand metrics," Phys. Lett. B 671, 493-495 (2009).

32 A. Herrero, and J. A. Morales-Lladosa, "Painlevé-Gullstrand synchronizations in spherical symmetry," Classical and Quantum Gravity 27, 175007 (2010).

33 E. Malec, and N. Ó Murchadha, "General spherically symmetric constant mean curvature foliations of the Schwarzschild solution," Phys. Rev. D 80, 024017 (2009). 
34 J. R. Oppenheimer, and H. Snyder, "On Continued Gravitational Contraction," Phys. Rev. 56, 455-459 (1939).

35 J. J. Ferrando, and J. A. Sáez, "An intrinsic characterization of spherically symmetric spacetimes," Classical and Quantum Gravity 27, 205024 (2010).

36 J. Plebański, and A. Krasiński, An Introduction to General Relativity and Cosmology (Cambridge University Press, Cambridge, 2006).

37 L. I. Petrich, S. L. Shapiro, and S. A., Teukolsky, "Oppenheimer-Snyder collapse in polar time slicing," Phys. Rev. D 33, 2100-2110 (1986).

38 E. Gourgoulhon, Ph. D. Thesis, Université de Paris VII (1992).

39 J. V. Romero, J. M. Ibáñez, J. M. Martí, and J. A. Miralles, "A New Spherically Symmetric General Relativistic Hydrodynamical Code," Astrophys. J. 462, 839 (1996).

40 I. Cordero-Carrión, J. M. Ibáñez, and J. A. Morales-Lladosa, "On the local existence of maximal slicings in spherically symmetric spacetimes," J. Phys.: Conf. Ser. 228, 012055 (2010).

41 E. Zauderer, Partial differential equations of applied mathematics, Second edition. (John Wiley \& Sons, New York, 1989).

42 M. Abramowitz, and I. A. Stegun, Handbook of mathematical functions, p. 556, (Dover Publications, Inc., New York, 1965).

43 P. Papadopoulos, and J. A. Font, "Relativistic hydrodynamics around black holes and horizon adapted coordinate systems," Phys. Rev. D 58, 024005 (1998).

44 D. Brill, "Maximal surfaces in closed and open spacetimes," in Proceedings of the 1st Marcel Grossmann meeting on general relativity. Edited by R. Ruffini (NorthHolland Pub. Comp., Amsterdam, 1977) pp. 193-206.

45 D. Brill, and F. Flaherty, "Isolated Maximal surfaces in Spacetime," Commun. Math. Phys. 50, 157-165 (1976). 